\title{
La Sostenibilidad en los grados de Educación Primaria de Andalucía
}

\author{
Lucía Rodríguez Pérez \\ Facultad de Ciencias de la Educación. Universidad de Cádiz. España. \\ lucia.rodriguezperez@alum.uca.es \\ ORCID: https://orcid.org/0000-0002-1622-8013
}

\begin{abstract}
Esther García González
Departamento de Didáctica, Área de Didáctica de las Ciencias Experimentales, Facultad de Educación. Universidad de Cádiz. España. esther.garcia@uca.es

ORCID: https://orcid.org/0000-0003-0995-9798
\end{abstract}

[Recibido: 24 mayo 2021. Revisado: 3 junio 2021. Aceptado: 9 diciembre 2021]

Resumen: Con el propósito de conocer la presencia de la Sostenibilidad en la formación de docentes, se ha realizado una investigación cualitativa en los grados de Educación Primaria en Andalucía. Se han realizado un análisis de las Memorias de grado, concretamente las dimensiones de los objetivos, contenidos, competencias, metodologías y sistemas de evaluación, siendo estos los elementos curriculares, los cuales se han valorado en forma de niveles que recogen criterios relacionados con la Sostenibilidad. Los resultados del estudio muestran que la Sostenibilidad difiere entre las universidades, ya que no existe un nivel homogéneo para todos los elementos curriculares. Sin embargo, todas la incluyen en sus Memorias, unas con más presencias que otras. En cuanto a objetivos, solo en Cádiz, Huelva, Málaga y Sevilla se incluye la Sostenibilidad. Con respecto a los contenidos, todas las universidades a excepción de Jaén y Málaga la incluyen. En relación con las competencias, las ocho universidades se establecen en el máximo nivel. Por su parte, en la metodología, ninguna universidad contempla la Sostenibilidad. Y, por último, solo en Sevilla se aprecia la presencia de la Sostenibilidad en el sistema de evaluación de una asignatura curricular. Tras lo recopilado, queda patente que la Sostenibilidad queda todavía lejos de establecerse de una manera más profunda en las Memorias de los grados en Educación Primaria del ámbito andaluz, como es el caso de estas ya analizadas, y que no solamente ha de quedarse en el terreno de la formación de maestros, sino que ha de incluirse en la formación de futuros profesionales de otras áreas.

Palabras clave: Sostenibilidad; educación primaria; maestros; universidad.

\section{Sustainability in Andalucía's Primary Education Grades}

Abstract: In order to find out about the presence of sustainability in teacher training, qualitative research has been carried out on Primary Education degrees in Andalucía. An analysis was made of the degree reports, specifically the dimensions of the objectives, contents, competences, methodologies and evaluation systems, these being the curricular elements, which have been evaluated in the form of levels that include criteria related to sustainability. The results of the study show that Sustainability differs between universities, as there is no homogeneous level for all curricular elements. However, all of them include it in their reports, some with more presence than others. In terms of objectives, only Cadiz, Huelva, Malaga and Seville include sustainability. With regard to content, all the universities except Jaén and Málaga include it. In relation to competences, all eight universities set the competences at the highest level. In terms of methodology, none of the universities include sustainability. Finally, only in Seville is Sustainability included in the evaluation system of a curricular subject. After what has been compiled, it is clear that sustainability is still far from being established in a more in-depth manner in the reports of the primary education degrees in Andalusia, as is the case of those already analysed, and that it should not only remain in the field of teacher training, but should also be included in the training of future professionals in other areas.

Keywords: Sustainability; Primary Education; teachers; University.

${ }^{1}$ Se utilizará el uso genérico del masculino en el lenguaje de este texto

Revista de Educación Ambiental y Sostenibilidad

Universidad de Cádiz. ISSN: 2659-708X

http://dx.doi.org/10.25267/Rev_educ_ambient_sostenibilidad.2021.v3.i2.2301

https://revistas.uca.es/index.php/ReAys/index 
Para citar este artículo: Rodríguez-Pérez, L. \& García-González, E. (2021) La Sostenibilidad en los grados de Educación Primaria de Andalucía. Revista de Educación Ambiental y Sostenibilidad 3(2), 2301. doi: 10.25267/Rev_educ_ambient_sostenibilidad.2021.v3.i2.2301

\section{La Sostenibilidad en la formación de maestros}

Ante los problemas socio-ambientales que encontramos actualmente en nuestro planeta, la educación es considerada una vía esencial para abordarlos (Álvarez, 2004), puesto que es un instrumento indispensable que provee de conocimientos, valores y actitudes que pueden hacer posible el cambio global que nuestra sociedad necesita. Es necesario actuar cuanto antes para que las nuevas generaciones puedan conseguir lo que las anteriores no han logrado (ORR, 2004 citado en Torres-Porras \& Arrebola, 2018), mejorar la situación y asegurar un futuro a nuestros descendientes.

Este estudio pretende analizar la presencia de la sostenibilidad en el Grado en Educación Primaria, encargado de formar a los futuros maestros, de las ocho universidades públicas de Andalucía, haciendo uso de una metodología cualitativa mediante el análisis de documentos donde se desarrolla el sentido de esta investigación.

Atendiendo al propósito indicado, tomamos la consideración de la Sostenibilidad como elemento clave de los procesos formativos, ya que esta persigue la búsqueda de una calidad ambiental, la justicia social y una economía equitativa y viable a largo plazo (CADEP-CRUE, 2012), implicando aspectos como el comportamiento ético, la protección del medio ambiente, los derechos humanos o la producción y el consumo responsables, entre otros aspectos claves para la formación de maestros.

Atendiendo a las consideraciones de Macedo (2005), harían falta una serie de acciones en el terreno educativo que transformen las concepciones, los hábitos y las perspectivas que el ser humano tiene predeterminadas, de manera que estén enfocadas hacia la participación ciudadana, las políticas medioambientales y hacia una sociedad sostenible.

Aunado a la situación y en un intento más por dar solución a las problemáticas ambientales, los Objetivos del Desarrollo Sostenible (ODS en adelante) marcan un conjunto de objetivos globales para erradicar los problemas que afectan al planeta. Para alcanzar las metas propuestas, es imprescindible que pongan de su parte, tanto gobiernos como ciudadanos, de modo que puedan lograrse para el año 2030. La llamada Agenda 2030 recoge los 17 ODS y señala los aspectos más importantes que permiten trabajar a los diferentes países bajo sus diferentes realidades, capacidades y niveles de desarrollo (UNESCO, 2017b).

Entre ellos encontramos el ODS 4 (Educación de calidad) donde aparecen una serie de objetivos enfocados a reorientar la educación y el aprendizaje para la adquisición de conocimientos, habilidades y valores que permitan contribuir a un futuro sostenible (Geli et al., 2019; Murga-Menoyo y Bautista-Cerro, 2019). De esta forma la Agenda 2030 apuesta por una educación inclusiva, equitativa y de calidad (De la Rosa et al., 2019), estableciendo cambios que han de producirse para conseguir el Desarrollo Sostenible, de modo que se promueva la responsabilidad y la conciencia social de la ciudadanía, así como la formación necesaria para que las personas tomen decisiones acordes con la calidad de vida y la Sostenibilidad (González 2003, citado en De la Rosa 
et al., 2019). En este proceso, la universidad es un organismo fundamental en la formación de personas y pilar esencial para el afrontamiento de los retos sociales (Kioupi y Voulvoulis, 2019 citado en García-González et al., 2020a), por lo que la Agenda cuenta con el respaldo de esta para crear una cultura enfocada a la Responsabilidad Social, contribuyendo así a la transformación social.

En este sentido, la importancia de sostenibilizar el currículum del Grado en Educación Primaria, tema principal que atañe esta investigación, incide en la formación de los futuros maestros para promover la Sostenibilidad y las prácticas relacionadas con ella. Es preciso, por tanto, hacer un esfuerzo por incorporar la Educación para la Sostenibilidad (en adelante ES) como un objetivo clave en este contexto.

La finalidad de formar a los maestros en Sostenibilidad es que estos a su vez formen académicamente a sus alumnos para que desarrollen un pensamiento crítico y competencias pertinentes (UNESCO 2017a), ya que es crucial para abordar la complejidad de la problemática socio-ambiental.

La Sostenibilidad Curricular en el ámbito de la formación de maestros no consiste solo en incluir contenidos ambientales en los currículums, sino también en proporcionar un cambio de mentalidad en el alumnado, desde actitudes favorables hasta metodologías adecuadas (Cardeñoso et al., 2013).

En relación con la universidad, institución encargada de formar a los futuros maestros, la UNESCO (1998, citado en Cardeñoso et al., 2013) estipuló que estas tienen la función de liderazgo en las formas de educación inter y transdisciplinarias, de modo que aporten soluciones para los problemas relacionados con la Sostenibilidad. Para ello han de integrar aspectos de la educación y el aprendizaje, de valores y principios que puedan ser satisfactorios para las necesidades actuales del planeta sin que ello implique un peligro para el futuro. Integrar una ES en todos los ámbitos educativos, que incide en la formación de los ciudadanos y profesionales, es una función que debería desempeñar la universidad como gran institución e influyente para todas aquellas personas que formen parte de ella (Geli, et al., 2019).

En España, la especificación de competencias clave en Sostenibilidad dentro de los currículums es una normativa impuesta en las universidades para sus planes de estudio en el proceso de armonización europea de los estudios superiores (Vilches \& Gil, 2012). Es por esta razón por la que se ha promovido la Sostenibilidad en los Grados y Postgrados universitarios, introduciendo esos conocimientos, valores y criterios de los que hablábamos antes. (Aznar-Minguet \& Ull-Solís, 2019; Geli et al., 2019).

Para Cebrián \& Junyent (2014), existen una serie de competencias que han de tener los maestros con respecto a la Sostenibilidad en todos los niveles y ámbitos educativos. Estas competencias se basan en el Informe Delors (1996) presentando tres características: un enfoque holístico e integrador, una visión del cambio y el objetivo de lograr la transformación.

Según el Informe de la UNESCO "La Educación encierra un tesoro" (Delors, 1996 citado en Albareda-Tiana \& Gonzalvo, 2013), las competencias para alcanzar el Desarrollo Sostenible en la educación superior serían: 
- Cognitivas (Saber)

- Metodológicas (saber hacer)

- Actitudinales (saber ser y valorar)

De igual forma, Cebrián \& Junyent (2014) exponían que el grupo de trabajo en Formación Inicial del Profesorado en el IV Congreso Internacional en Educación Ambiental "Tblisiplus30", definió las competencias que tendrían que ser consideradas en la formación del profesorado (citado en Hopkins \& Ravindranath, 2007):

- La reflexión ética sistemática sobre los valores e intereses relativos a las relaciones humanas y al cuidado de la naturaleza. Considerar de manera integral la participación activa y la co-construcción de las prácticas educativas y de la ES en la sociedad.

- La capacidad de apoyar y gestionar la diversidad cultural y social.

- La capacidad para identificar las conexiones entre la dimensión sociocultural, la ecológica y la económica del medio ambiente.

- La capacidad para identificar las conexiones entre las condiciones ambientales locales y globales y dibujar inferencias significativas en los contextos de formación.

- La confianza e interés en compartir experiencias, conocimiento, capacidades y valores con los demás.

Sobre las bases de las ideas expuestas las cuestiones de investigación que nos planteamos son las siguientes:

- ¿Incluyen los Grados en Educación Primaria de las universidades andaluzas aspectos relacionados con la Sostenibilidad en sus Memorias?

- ¿Cuáles de dichos aspectos aparecen en las asignaturas?

- ¿Qué competencias en Sostenibilidad están presentes?

- ¿Cuáles son los objetivos que hacen referencia a la Sostenibilidad?

- ¿Qué contenidos en Sostenibilidad están presentes?

- ¿Están las competencias, objetivos y contenidos reflejados en la metodología propuesta? ¿y en la evaluación?

En este sentido, el estudio tiene como objetivo caracterizar el grado de inclusión de la Sostenibilidad en los grados Educación Primaria de las ocho universidades públicas andaluzas.

\section{Metodología}

La propuesta de la presente investigación es analizar si están recogidas, y cómo, cuestiones referidas a la Sostenibilidad en el conjunto de los planes de estudio, analizando los elementos que configuran cada una de las asignaturas que componen dichos planes.

Se propone el enfoque cualitativo de corte interpretativo, dada la naturaleza del fenómeno de estudio. La investigación cualitativa se orienta hacia la comprensión de situaciones únicas, centrándose en la búsqueda de significado y de sentido que se les 
conceden a las situaciones, observando y experimentando fenómenos o experiencias de individuos o grupos sociales (Gómez \& Roquet, 2009, p.47).

En cuanto a la técnica, se ha optado por la observación semisistemática, considerado el análisis de contenido como método más conveniente, de manera que se analizarán las propuestas recogidas en el conjunto de documentos seleccionados. Este método se ubica en el ámbito de lo descriptivo y pretende detectar los componentes básicos de un determinado fenómeno a través de su contenido (López Noguero, 2002).

Asimismo, la muestra seleccionada es intencionada y reducida, al tratarse de una primera aproximación a este campo. Se han seleccionado las Memorias de Grado de Educación Primaria de las universidades de Almería, Cádiz, Córdoba, Granada, Huelva, Jaén, Málaga y Sevilla. En ellas, se han analizado minuciosamente las asignaturas de cada uno de dichos grados, suponiendo 59 en el Grado de Almería, 59 en el de Cádiz, 54 en Córdoba, 51 en Granada, 59 en Huelva, 67 en Jaén, 62 en Málaga y 52 en Sevilla. En total ha supuesto 463 asignaturas analizadas.

Para el tratamiento de los datos, tomamos como dimensiones de análisis los elementos curriculares que constituyen el plan de estudio de las Memorias de Grado especificadas:

- Dimensión 1: objetivos

- Dimensión 2: contenidos

- Dimensión 3: competencias

- Dimensión 4: metodologías

- Dimensión 5: sistemas de evaluación

Estas dimensiones están relacionadas con las cuestiones de investigación formuladas y surgen de la teoría, predefinidas y elegidas para identificar la presencia de la Sostenibilidad en esta titulación universitaria, ya que son los pilares en los que se sustentan los procesos de enseñanza-aprendizaje de cualquier disciplina.

A continuación, procedemos a definir cada dimensión en materia de Sostenibilidad:

- Objetivos: tomando como referencia los ODS, son los logros a los que el mundo debería aspirar (Sachs \& Vernis, 2015), siendo los referentes relativos a los logros que el alumnado debe alcanzar al finalizar el proceso educativo, como resultado de experiencias enseñanza-aprendizaje intencionalmente planificadas para tal fin (Ley Orgánica, 8/2013).

- Contenidos: aquellos conocimientos que se ordenan en asignaturas y que trabajan el medio ambiente, la capacidad de los estudiantes para solucionar problemas socio-ambientales desde su tratamiento en el ámbito local y global (Barrón et al., 2010).

- Competencias: conjunto complejo e integrado de conocimientos, habilidades, actitudes y destrezas (Erice, 2004) que permitan resolver problemas socioambientales mediante un pensamiento crítico (Rieckman, 2012 citado en Albareda-Tiana \& Gonzalvo 2013).

- Metodología: conjunto de estrategias, procedimientos y acciones organizadas y planificadas por el profesorado (Ley Orgánica, 8/2013), requiriendo un diseño 
de material que se concrete en una guía de educación universitaria sostenible aplicable a todas las titulaciones (Ruiz-Rico, 2016).

- Sistemas de evaluación: son el referente específico para evaluar el aprendizaje del alumnado y describen aquello que se quiere valorar y que alumnado debe lograr en materia de Sostenibilidad, tanto en conocimientos como en competencias, respondiendo a lo que se pretende conseguir en cada asignatura (Ley Orgánica, 8/2013).

Una vez definidas las dimensiones, se realizó una clasificación por guías de las unidades de información (UI en adelante) extraídas en el análisis de los datos, donde emergen los indicadores, establecidos en forma de niveles, siendo el 0 el más bajo y el 3 el más alto. Estos hacen referencia a la Sostenibilidad y su presencia en relación a cada dimensión. En este procedimiento, la fundamentación teórica fue tomada como referencia y dio sentido a los indicadores. La rúbrica empleada para reflejar el sistema de análisis (Tabla 1) ha sido el instrumento empleado, el cual ha sido expuesto a una valoración de interjueces dentro del grupo de investigación, y apoyado por la literatura (Poza-Vilches et al., 2021; Azcárate et al., 2012; García-González et al., 2020a)

Tabla 1: Sistema de análisis. Fuente: elaboración propia

\begin{tabular}{|c|c|c|c|c|}
\hline & Nivel 0 & Nivel 1 & Nivel 2 & Nivel 3 \\
\hline Dimensión 1 & $\begin{array}{l}\text { Los objetivos } \\
\text { presentes en las } \\
\text { memorias no } \\
\text { hacen referencia } \\
\text { a temas } \\
\text { relacionados con } \\
\text { la Sostenibilidad }\end{array}$ & $\begin{array}{lr}\text { Los objetivos } \\
\text { presentes en las } \\
\text { memorias hacen } \\
\text { referencia } \\
\text { temas } \\
\text { relacionados } \\
\text { la Sostenibilidad } \\
\text { solo } \\
\text { asignaturas de } \\
\text { didáctica de las } \\
\text { ciencias } \\
\text { experimentales }\end{array}$ & $\begin{array}{l}\text { Los objetivos } \\
\text { presentes en las } \\
\text { memorias hacen } \\
\text { referencia } \\
\text { temas a } \\
\text { relacionados con } \\
\text { la Sostenibilidad } \\
\text { en contenidos, } \\
\text { competencias, } \\
\text { metodologías y } \\
\text { sistemas } \\
\text { evaluación de } \\
\text { asignaturas de } \\
\text { didáctica de las } \\
\text { ciencias sociales o } \\
\text { didáctica de la } \\
\text { lengua }\end{array}$ & 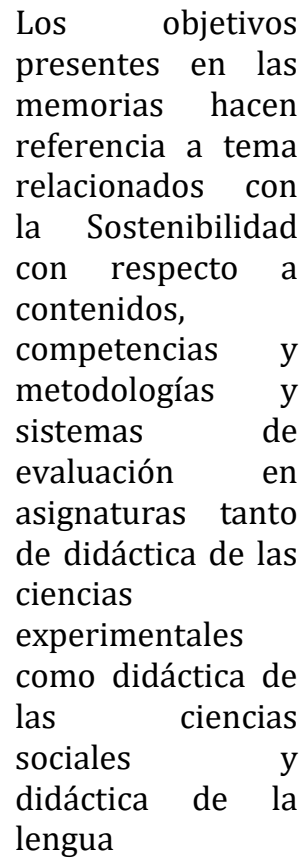 \\
\hline Dimensión 2 & $\begin{array}{l}\text { La Sostenibilidad } \\
\text { no es un } \\
\text { contenido de las } \\
\text { asignaturas }\end{array}$ & $\begin{array}{lr}\text { La Sostenibilidad } \\
\text { está presente } \\
\text { como contenido } \\
\text { solo r en } \\
\text { asignaturas de } \\
\text { didáctica de las } \\
\text { ciencias } \\
\text { experimentales }\end{array}$ & $\begin{array}{l}\text { La Sostenibilidad } \\
\text { está presente } \\
\text { como contenido } \\
\text { en asignaturas de } \\
\text { didáctica de las } \\
\text { ciencias sociales o } \\
\text { didáctica de la } \\
\text { lengua }\end{array}$ & $\begin{array}{l}\text { La Sostenibilidad } \\
\text { está presente } \\
\text { como contenido en } \\
\text { asignaturas tanto } \\
\text { de didáctica de las } \\
\text { ciencias } \\
\text { experimentales } \\
\text { como didáctica de } \\
\text { las ciencias } \\
\text { sociales de } \\
\text { didáctica de la } \\
\text { lengua }\end{array}$ \\
\hline
\end{tabular}


Tabla 1: Continuación

\begin{tabular}{|c|c|c|c|c|}
\hline & Nivel 0 & Nivel 1 & Nivel 2 & Nivel 3 \\
\hline Dimensión 3 & $\begin{array}{l}\text { No se } \\
\text { contemplan } \\
\text { conocimientos, } \\
\text { habilidades, } \\
\text { actitudes y } \\
\text { destrezas } \\
\text { relacionadas con } \\
\text { la Sostenibilidad }\end{array}$ & $\begin{array}{l}\text { Se contemplan } \\
\text { conocimientos, } \\
\text { habilidades, } \\
\text { actitudes } \\
\text { destrezas } \\
\text { relacionadas con } \\
\text { la Sostenibilidad } \\
\text { solo en } \\
\text { asignaturas de } \\
\text { didáctica de las } \\
\text { ciencias } \\
\text { experimentales }\end{array}$ & $\begin{array}{l}\text { Se contemplan } \\
\text { conocimientos, } \\
\text { habilidades, } \\
\text { actitudes } \\
\text { destrezas } \\
\text { relacionadas con } \\
\text { la Sostenibilidad } \\
\text { en asignaturas de } \\
\text { didáctica de las } \\
\text { ciencias sociales o } \\
\text { didáctica de la } \\
\text { lengua }\end{array}$ & $\begin{array}{l}\text { Se contemplan } \\
\text { conocimientos, } \\
\text { habilidades, } \\
\text { actitudes } \\
\text { destrezas } \\
\text { relacionadas con la } \\
\text { Sostenibilidad en } \\
\text { asignaturas tanto } \\
\text { de didáctica de las } \\
\text { ciencias } \\
\text { experimentales } \\
\text { como didáctica de } \\
\text { las ciencias sociales } \\
\text { y didáctica de la } \\
\text { lengua }\end{array}$ \\
\hline Dimensión 4 & $\begin{array}{l}\text { Las metodologías } \\
\text { presentes en las } \\
\text { memorias no se } \\
\text { corresponden } \\
\text { con } \\
\text { metodologías } \\
\text { que promueven } \\
\text { la integración de } \\
\text { la Sostenibilidad }\end{array}$ & $\begin{array}{l}\text { Las metodologías } \\
\text { presentes en las } \\
\text { memorias } \\
\text { promueven la } \\
\text { integración de la } \\
\text { Sostenibilidad } \\
\text { solo en } \\
\text { asignaturas de } \\
\text { didáctica de las } \\
\text { ciencias } \\
\text { experimentales }\end{array}$ & $\begin{array}{l}\text { Las metodologías } \\
\text { presentes en las } \\
\text { memorias } \\
\text { promueven la } \\
\text { integración de la } \\
\text { Sostenibilidad en } \\
\text { asignaturas de } \\
\text { didáctica de las } \\
\text { ciencias sociales o } \\
\text { didáctica de la } \\
\text { lengua }\end{array}$ & $\begin{array}{l}\text { Las metodologías } \\
\text { presentes en las } \\
\text { memorias } \\
\text { promueven la } \\
\text { integración de la } \\
\text { Sostenibilidad en } \\
\text { asignaturas tanto } \\
\text { de didáctica de las } \\
\text { ciencias } \\
\text { experimentales } \\
\text { como didáctica de } \\
\text { las ciencias sociales } \\
\text { y didáctica de la } \\
\text { lengua }\end{array}$ \\
\hline Dimensión 5 & $\begin{array}{l}\text { Los sistemas de } \\
\text { evaluación } \\
\text { presentes en las } \\
\text { memorias no } \\
\text { valoran lo que el } \\
\text { alumnado debe } \\
\text { lograr en materia } \\
\text { de Sostenibilidad }\end{array}$ & $\begin{array}{l}\text { Los sistemas de } \\
\text { evaluación } \\
\text { presentes en las } \\
\text { memorias } \\
\text { valoran lo que el } \\
\text { alumnado debe } \\
\text { lograr en materia } \\
\text { de Sostenibilidad } \\
\text { solo en } \\
\text { asignaturas las } \\
\text { didáctica de la } \\
\text { ciencias } \\
\text { experimentales }\end{array}$ & $\begin{array}{l}\text { Los sistemas de } \\
\text { evaluación } \\
\text { presentes en las } \\
\text { memorias valoran } \\
\text { lo que el } \\
\text { alumnado debe } \\
\text { lograr en materia } \\
\text { de Sostenibilidad } \\
\text { en asignaturas de } \\
\text { didáctica de las } \\
\text { ciencias sociales o } \\
\text { didáctica de la } \\
\text { lengua }\end{array}$ & $\begin{array}{l}\text { Los sistemas de } \\
\text { evaluación } \\
\text { presentes en las } \\
\text { memorias valoran } \\
\text { lo que el alumnado } \\
\text { debe lograr en } \\
\text { materia de } \\
\text { Sostenibilidad en } \\
\text { asignaturas tanto } \\
\text { de didáctica de las } \\
\text { ciencias } \\
\text { experimentales } \\
\text { como didáctica de } \\
\text { las ciencias sociales } \\
\text { y didáctica de la } \\
\text { lengua }\end{array}$ \\
\hline
\end{tabular}

Para analizar cada UI, estas han sido asociadas a una dimensión y a un nivel del sistema de análisis una por una, mediante la lectura de las Memorias y anotando toda aquella información referente a la Sostenibilidad que se ajustara a los criterios de clasificación de los niveles expuestos en la rúbrica.

La codificación de las UI fue: 
- Memoria de Almería:

GUA_D_No: Guía universidad de Almería_Dimensión_Número UI

- Memoria de Cádiz:

GUC_D_No: Guía universidad de Cádiz_Dimensión_Número UI

- Memoria de Córdoba:

GUCO_D_No: Guía universidad de Córdoba_Dimensión_Número UI

- Memoria de Granada:

GUGR_D_No: Guía universidad de Granada _Dimensión_Número UI

- Memoria de Huelva:

GUH_D_No: Guía universidad de Huelva_Dimensión_Número UI

- Memoria de Jaén:

GUJ_D_No: Guía universidad de Jaén_Dimensión_Número UI

- Memoria de Málaga:

GUM_D_No: Guía universidad de Málaga_Dimensión_Número UI

- Memoria de Sevilla:

GUS_D_No: Guía universidad de Sevilla_Dimensión_Número UI

En cuanto a los criterios para asociar cada nivel a la dimensión de cada universidad, lo primero que se ha tenido en cuenta ha sido el tipo de asignatura, es decir, si procede de didáctica de las ciencias experimentales o de didáctica de las ciencias sociales. Por regla general, la Sostenibilidad suele aparecer con más frecuencia en asignaturas ligadas a las ciencias experimentales, por lo que el estudio se centra en detectar que realmente puede aparecer en asignaturas de disciplinas como las ciencias sociales o didáctica de la lengua en formas de objetivos, contenidos, competencias, metodologías o sistemas de evaluación Para ello, mostramos en la figura 1 la presencia de la Sostenibilidad cuando hablamos de las dimensiones, haciendo una comparativa con cada universidad y detectando en cuáles de ellas la Sostenibilidad aparece de una manera más o menos profunda, es decir, teniendo en cuenta los niveles que hemos establecido.

Estos niveles siguen el patrón establecido en la rúbrica expuesta, de manera que el Nivel 0 especifica que no existe la Sostenibilidad en ninguna de las asignaturas, el Nivel 1 que aparece en al menos una asignatura de Ciencias Experimentales, el Nivel 2 que aparece en al menos una asignatura de Ciencias Sociales o Didáctica de la lengua, y el Nivel 3 que aparece al menos en una asignatura de Ciencias Experimentales y en otra de Ciencias Sociales o Didáctica de la lengua.

\section{Resultados y discusión}

A continuación, se presentan los resultados obtenidos en el análisis de cada universidad, exponiéndolos gráficamente al final del discurso.

\section{Universidad de Almería}

En cuanto a los resultados obtenidos tras el análisis, en la Universidad de Almería (Figura 1), no se han encontrado objetivos que hagan referencia a la Sostenibilidad, por lo que esta dimensión se encuentra en el Nivel 0. En relación con los contenidos, han sido clasificados en el Nivel 2 al encontrarse un contenido en la asignatura 
Sociedad, Escuela y Democracia. Véase este contenido:

Las transformaciones de la sociedad actual: Sociedad de la información. Sociedad y relaciones de género. Sociedad y relaciones intergeneracionales. Sociedad multicultural. Sociedad de la exclusión. Sociedad y desarrollo sostenible. GUA_D2_1

Con respecto a las competencias, se han establecido en un Nivel 3 al contemplarse la Sostenibilidad en tres asignaturas: Didáctica de las Ciencias Naturales I (C4.5); Sociedad, escuela y democracia; y Sociología de la Educación y de la Familia (C3.4). Ambas competencias pertenecen a las específicas. Veamos un ejemplo de la C4.5:

4.5 - Reconocer la mutua influencia entre ciencia, sociedad y desarrollo tecnológico, así como las conductas ciudadanas pertinentes, para procurar un futuro sostenible. GUA_D3_1

Siguiendo en la misma línea, habiendo analizado la metodología de la memoria de Almería y no habiendo encontrado elemento alguno que promueva la Sostenibilidad en las actividades realizadas en las asignaturas, la situamos en un Nivel 0.

De la misma manera, los sistemas de evaluación presentes tampoco valoran lo que el alumnado debe saber en materia de Sostenibilidad, clasificándolo también en un Nivel 0.

\section{Universidad de Cádiz}

Tras el análisis realizado en la Memoria de Cádiz (Figura 1), podemos observar que los objetivos se encuentran en un Nivel 3, al aparecer dos en las siguientes asignaturas:

La educación ambiental como eje integrador:

Comprender la necesidad de la Educación Ambiental formal desde un punto de vista transversal como instrumento para la Sostenibilidad. GUC_D1_1.

Sociología de la Educación:

Conocer los debates sociales que afectan a la educación familiar y escolar: género, inmigración, violencia, inclusión/exclusión dentro de los grupos sociales y desarrollo sostenible. GUC_D1_2.

En cuanto a los contenidos, establecidos en el Nivel 1, en la asignatura La educación ambiental como eje integrador encontramos este:

Medio ambiente y problemática ambiental, el medio ambiente como sistema complejo. GUC_D2_2.

Con respecto a las competencias, hemos detectado tres de ellas en asignaturas tanto de Didáctica de las Ciencias Experimentales como en Didáctica de las Ciencias Sociales o Didáctica de la Lengua. Se trata de la competencia general CG23, la competencia transversal CT2 y la competencia específica CE5. Véase un ejemplo:

CT2. Sostenibilidad y compromiso ambiental. Uso equitativo, responsable y eficiente de los recursos. GUC_D3_1.

De acuerdo a nuestro sistema de análisis, al encontrar competencias relacionadas con la Sostenibilidad tanto en asignaturas de didáctica de las ciencias experimentales 
como en didáctica de las ciencias sociales, cabría clasificarlas en un Nivel 3.

Por último, en el caso de la metodología y los sistemas de evaluación, su Nivel 0 corresponde con la inexistencia de elementos que promuevan la Sostenibilidad, ni en las actividades realizadas ni en la valoración en cuanto a lo que el alumnado ha de saber en materia de Sostenibilidad.

\section{Universidad de Córdoba}

Atendiendo al análisis de la Memoria de Córdoba (Figura 1), hemos detectado que los objetivos se encuentran en un Nivel 0 , ya que ninguno hace referencia a temas relacionados con la Sostenibilidad en ninguna de las asignaturas analizadas.

En cuanto a los contenidos, por el contrario, se han clasificado en el máximo nivel, el 3 , ya que la Sostenibilidad está presente en asignaturas tanto de Didáctica de las Ciencias Experimentales como en Didáctica de las Ciencias Sociales y Didáctica de la Lengua.

De la asignatura de Ciencias Experimentales, Didáctica del Medio Ambiente:

Medio ambiente y desarrollo humano. Cuantificación de recursos, impactos y riesgos medioambientales en función del desarrollo personal, social y tecnológico. GUCO_D2_2.

De la asignatura de Ciencias Sociales, Europa y el Medio Ambiente:

Se pretende llevar al alumnado a ser competentes en la capacitación de su futuro alumnado como ciudadano europeo en su posicionamiento social, ambiental y económico. GUCO_D2_4.

Con respecto a las competencias, han sido clasificadas en un Nivel 3, ya que aparecen conocimientos, habilidades, actitudes y destrezas relacionadas con la Sostenibilidad en asignaturas de Didáctica de las Ciencias Experimentales y de Didáctica de las Ciencias Sociales o Didáctica de la Lengua. Estas competencias pertenecen a GUCO_D3_3 y son las competencias específicas CE9 y CE14, y las competencias modulares CM3.4, CM4.5 y CM2.12. Veamos un ejemplo:

CM2.12: Participar en la definición del proyecto educativo y en la actividad general del centro atendiendo a criterios de gestión de calidad y de sostenibilidad. GUCO_D3_5.

No obstante, al igual que en las dos memorias anteriores, la metodología y los sistemas de evaluación se han clasificado en un Nivel 0 , puesto que no se han encontrado elementos que promuevan la Sostenibilidad en las metodologías planteadas, ni tampoco se ha puesto en valor lo que el alumnado debe saber en materia de Sostenibilidad a la hora de la evaluación.

\section{Universidad de Granada}

El caso de la memoria de Granada es similar al de Córdoba. Podemos apreciar los resultados en la figura 1. Comenzando por los objetivos, al no hacer referencia alguna a la Sostenibilidad en las asignaturas del Grado, se encuentran en el Nivel 0.

Con respecto a los contenidos, estos han sido clasificados en el Nivel 3 al detectar la presencia de la Sostenibilidad como un contenido dentro de una asignatura de Didáctica de las Ciencias Experimentales y otro en una asignatura de Didáctica de las 
Ciencias Sociales. Véase el ejemplo de la asignatura Enseñanza de la Educación Física en la Educación Primaria:

Evolución, situación actual y desarrollo de las competencias. Enfoque crítico de la Educación Física Escolar para el siglo XXI: atención a la diversidad, ciudadanía y sostenibilidad. GUGR_D2_2.

Seguidamente, en el caso de las competencias, cabría destacar la presencia de tres de ellas que aparecen en numerosas asignaturas del currículum, tanto de Didáctica de las Ciencias Experimentales como Didáctica de las Ciencias Sociales. Estas competencias serían la C9, la CDM3.4 y la CDM4.5, correspondiéndose la primera a las competencias específicas y las dos últimas a las competencias específicas de formación básica. Véase un ejemplo:

C9 Valorar la responsabilidad individual y colectiva en la consecución de un futuro sostenible. GUGR_D3_1.

Mientras tanto, la metodología y los sistemas de evaluación una vez más se encuentran en un Nivel 0 , al no haber referencia alguna de la Sostenibilidad en las actividades planteadas, ni tampoco en las evaluaciones.

\section{Universidad de Huelva}

En el análisis realizado en la memoria del Grado de Educación Primaria de Huelva (Figura 1), se han encontrado dos objetivos que hacen referencia a temas relacionados con la Sostenibilidad en la asignatura de "Didáctica de las Ciencias de la Naturaleza", el 08 y el 010. El primero de ellos se refiere a lo siguiente:

08: Reconocer la mutua influencia entre ciencia, sociedad y desarrollo tecnológico, así como las conductas ciudadanas pertinentes, para procurar un futuro sostenible. GUH_D1_2.

En relación con los contenidos, situados en el Nivel 2, mostramos el que encontramos en la asignatura de "Lengua extranjera (francés)":

4.- Desarrollo Sostenible, comercio justo. GUH_D2_1.

Del mismo modo, en lo que respecta a las competencias, las relacionadas con la Sostenibilidad presentes en la memoria de Huelva son cuatro, las específicas E23 y E29, la general G13 y la transversal CT16. Tomamos como ejemplo esta última:

CT6 - Promover, respetar y velar por los derechos humanos, la igualdad sin discriminación por razón de nacimiento, raza, sexo, religión, opinión u otra circunstancia personal o social, los valores democráticos, la igualdad social y el sostenimiento medioambiental. GUH_D3_2.

Estas competencias se encuentran tanto en asignaturas de Didáctica de las Ciencias Experimentales como en Didáctica de las Ciencias Sociales o Didáctica de la Lengua, por lo tanto, cabría situarlas en un Nivel 3.

Por último y, una vez más coincidiendo con las memorias analizadas anteriormente, la metodología y los sistemas de evaluación no cumplen con ninguno de los Niveles del 1 al 3 en los que se trabaje la Sostenibilidad a la hora de planificar actividades formativas y valoraciones de las asignaturas. 


\section{Universidad de Jaén}

Siguiendo en la misma línea, tras el análisis de la memoria de Jaén se han hecho las siguientes clasificaciones que podemos observar en la figura 1.

En cuanto a los objetivos, de la misma forma que en los Grados de Almería, Córdoba y Granada, se han clasificado en un Nivel 0 según nuestro sistema de análisis, al no encontrar ningún objetivo que haga referencia a temas relacionados con la Sostenibilidad en las asignaturas curriculares.

Con respecto a los contenidos, también se encuentran en un Nivel 0 , puesto que la Sostenibilidad aparece como contenido en ninguna de las asignaturas que componen el Grado.

Sin embargo, las competencias, a diferencia de estas dos últimas dimensiones de análisis, se han clasificado en el Nivel 3 al detectar que en las competencias básicas y generales C.F.B.23 y C.F.D.D.5 se contemplan conocimientos, habilidades, actitudes y destrezas relacionadas con la Sostenibilidad en asignaturas tanto de Didáctica de las Ciencias Experimentales como en Didáctica de las Ciencias Sociales. Véase el ejemplo:

C.F.D.D.5 - Reconocer la mutua influencia entre ciencia, sociedad y desarrollo tecnológico, así como las conductas ciudadanas pertinentes, para procurar un futuro sostenible. GUJ_D3_2.

Por último, la metodología y los sistemas de evaluación se corresponde, una vez más, con el Nivel 0 del sistema de análisis diseñado, puesto que las metodologías presentes en las memorias no se corresponden con metodologías que promueven la integración de la Sostenibilidad, del mismo modo que tampoco se valora lo que el alumnado debe lograr en materia de Sostenibilidad.

\section{Universidad de Málaga}

Tras el análisis de la memoria de Málaga (Figura 1), los objetivos que aparecen propuestos para la titulación de Educación Primaria marcan el propósito de que los aprendizajes se alcen lo más alto posible en la dirección que marcan las competencias generales y específicas de cada asignatura. Por esta razón, al igual que veremos más adelante con las competencias, los objetivos en este caso estarán clasificados en un Nivel 3.

En cuanto a los contenidos, a diferencia de los objetivos y las competencias, han de ser situados en el Nivel 0 al no encontrarse la Sostenibilidad como un contenido en ninguna de las asignaturas que conforman el Grado.

Sin embargo, como hemos mencionado anteriormente, las competencias, al igual que los objetivos, están clasificadas en un Nivel 3, pues ambas dimensiones de análisis van de la mano, encontrándose en asignaturas tanto de Didáctica de las Ciencias Experimentales como de Didáctica de las Ciencias Sociales. En el caso de las competencias, se han detectado tres que hacen ahínco a la Sostenibilidad, las competencias específicas CE 3.3.2, CE 4.1.4 y CE 2.9. Veamos el ejemplo:

CE 4.1.4 - Valorar la trascendencia de las ciencias como un hecho cultural y la necesidad de la alfabetización científica y tecnológica de la ciudadanía, reconociendo y valorando la mutua influencia entre ciencia, sociedad y desarrollo tecnológico, así como las conductas ciudadanas 


\section{pertinentes para procurar un futuro sostenible. GUM_D3_2.}

Con respecto a la metodología empleada, al igual que ocurre en las memorias de las anteriores universidades expuestas, las situaremos en un Nivel 0 , puesto que no se especifica nada relacionado con la Sostenibilidad en las actividades formativas que se llevan a cabo. Sin embargo, si analizamos las siguientes líneas de la Memoria del Grado en Educación Primaria de Málaga, la Sostenibilidad podría trabajarse siguiendo la organización y los métodos adecuados, según los objetivos y competencias propuestos:

La metodología implica la gestión del tipo de relaciones que se fomentan en el aula, de los distintos recursos educativos que se ponen a disposición del alumnado, de las formas de comunicación que se establecen, de las actividades formativas que se diseñan y desarrollan, de los modos de evaluación que se proponen, de la selección y organización de los tópicos fundamentales de los contenidos que se abordan. (p.42). GUM_D4_1.

Por último, en lo que respecta a los sistemas de evaluación, ocurre lo mismo que con la metodología, al no valorarse la Sostenibilidad explícitamente con respecto a lo que el alumnado debe adquirir al finalizar una asignatura. Pese a ello, tal como hacíamos alusión en la metodología, si observamos uno de los criterios, como es el caso del número 6, se puede apreciar una forma válida de evaluar cualquier disciplina, conocimiento, habilidad o destreza.

6. Valoración del Portafolios Criterios de evaluación:

- Capacidad para localizar y seleccionar información relevante.

-Capacidad para argumentar la importancia de la información seleccionada en el desarrollo de su aprendizaje.

- Capacidad para comunicar de forma lógica y ordenada el desarrollo de su aprendizaje.

- Capacidad para usar la teoría como herramienta conceptual al servicio de un análisis profundo, riguroso, y original de los aspectos seleccionados. GHM_D5_1.

A pesar de ello, en base a nuestro sistema de análisis, el sistema de evaluación estaría dentro del Nivel 0 .

\section{Universidad de Sevilla}

Como podemos observar en la figura 1 de la Memoria de Sevilla, hemos clasificado los objetivos en un Nivel 3 de nuestro sistema de análisis, puesto que se han encontrado dos objetivos que hacen referencia a temas relacionados con la Sostenibilidad con respecto a contenidos, competencias y metodologías y sistemas de evaluación en asignaturas tanto de Didáctica de las Ciencias Experimentales como Didáctica de las Ciencias Sociales. Ambos de estos objetivos hacen alusión a la competencia específica EP15 y a las competencias modulares M22 y M28.

Siguiendo en la misma línea, pasamos a los contenidos. Estos se han clasificado dentro del Nivel 1, puesto que se ha detectado que la Sostenibilidad está presente como un solo contenido en una sola asignatura, "Didáctica de las Ciencias Experimentales", cuyo contenido del BLOQUE III expone: 


\section{Enseñanza de las ciencias y sostenibilidad. GUS_D2_1.}

En lo que respecta a las competencias, como veníamos diciendo anteriormente a la hora de clasificar a los objetivos, se ha detectado la relación entre estos últimos y las competencias EP15, M22 y M28. Por ello, se han clasificado las competencias en el Nivel 3, puesto que tratan conocimientos, habilidades, actitudes y destrezas relacionadas con la Sostenibilidad en asignaturas tanto de Didáctica de las Ciencias Experimentales como Didáctica de las Ciencias Sociales. Veamos un ejemplo significativo del contenido de la siguiente competencia:

EP.15 Conocer las funciones, posibilidades y limitaciones de la educación para afrontar las responsabilidades sociales, promoviendo alternativas que den respuestas a dichas necesidades, en orden a la consecución de un futuro solidario y sostenible. GUS_D3_1.

Continuando con la metodología, al igual que ocurre en todas las memorias ya expuestas, la de Sevilla también se clasifica en un Nivel 0 al no especificar nada relacionado con la Sostenibilidad en las actividades formativas que se llevan a cabo en las asignaturas curriculares.

Y, por último, los sistemas de evaluación que, a diferencia de los analizados en las memorias anteriores, en Sevilla se han clasificado en un Nivel 2 al encontrar un criterio de evaluación de la asignatura de "Didáctica de las Matemáticas" que dice así:

La sostenibilidad (impacto social y medio ambiental desde una perspectiva integrada y sistémica). GUS_D5_1.

Una vez expuestos los resultados, veamos representativamente los niveles en los que están clasificadas las dimensiones de análisis por cada universidad:

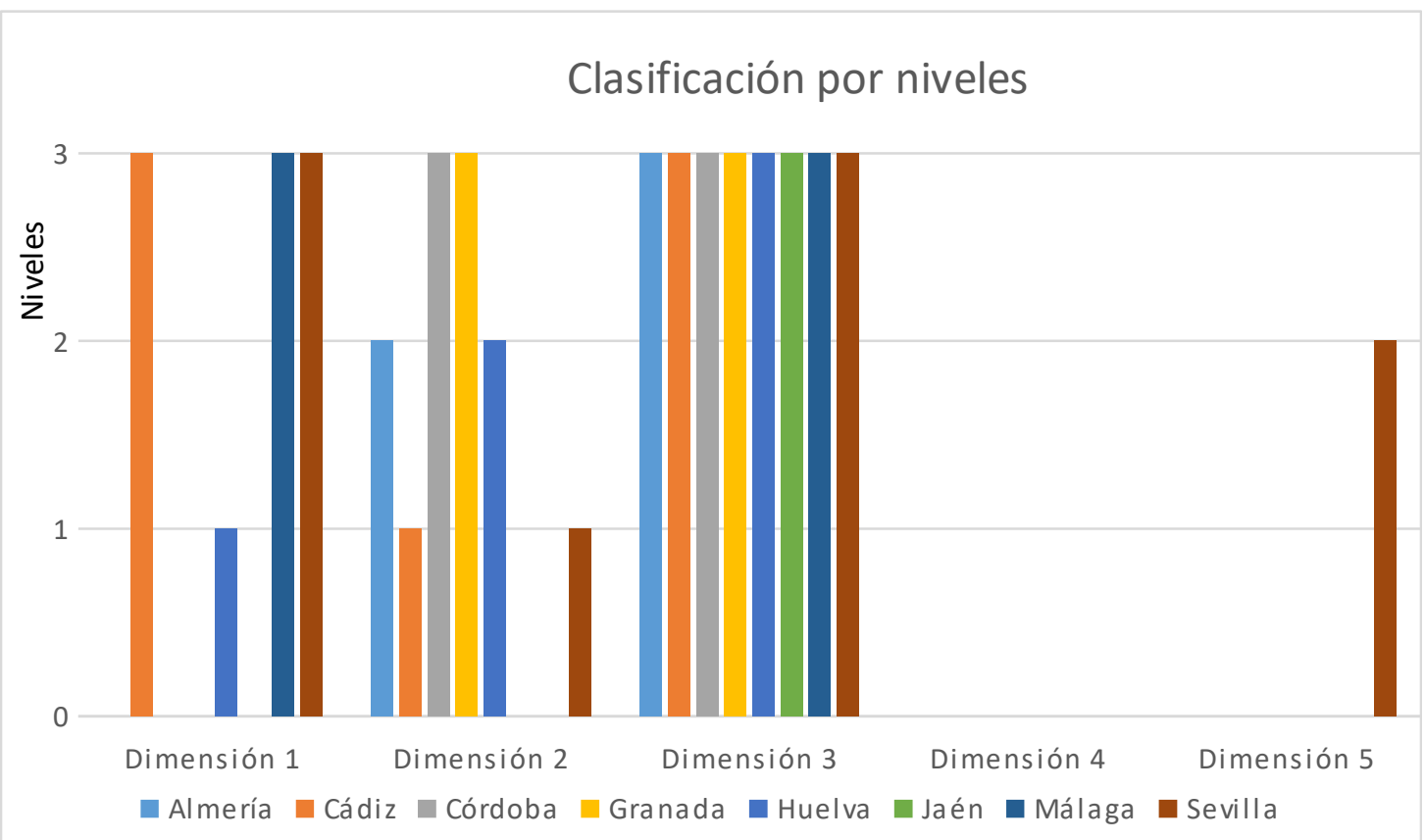

Figura 1: Clasificación de las universidades en niveles. Fuente: elaboración propia.

A continuación, mostramos la Tabla 2, donde podemos ver reflejadas todas las UI codificadas que hemos extraído de las Memorias. 
Tabla 2: Unidades de información. Fuente: elaboración propia

\begin{tabular}{|c|c|c|}
\hline & $\begin{array}{l}\text { Didáctica de las Ciencias } \\
\text { Experimentales }\end{array}$ & $\begin{array}{l}\text { Didácticas de las Ciencias } \\
\text { Sociales o de la Lengua }\end{array}$ \\
\hline Universidad Almería & C 4.5: GUA_D3_1 & $\begin{array}{l}\text { GUA_D2_1 } \\
\text { C 3.4: GUA_D3_2 }\end{array}$ \\
\hline Universidad Cádiz & $\begin{array}{l}\text { GUC_D1_1 } \\
\text { GUC_D2_1 } \\
\text { CT2: GUC_D3_1, CE5: GUC_D3_2 }\end{array}$ & $\begin{array}{l}\text { GUC_D1_2 } \\
\text { CG23:GUC_D3_3 }\end{array}$ \\
\hline Universidad Córdoba & $\begin{array}{l}\text { GUCO_D2_1, GUCO_D2_2 } \\
\text { CE9:GUCO_D3_1, } \\
\text { CM4.5GUCO_D3_2, } \\
\text { CE14:GUC0_D3_3 }\end{array}$ & $\begin{array}{l}\text { GUCO_D2_3, GUCO_D2_4 } \\
\text { CE9: GUCO_D3_1,CM4.5: } \\
\text { GUCO_D3_2, CE14: GUCO_D3_3, } \\
\text { CM4.3: GUCO_D3_4, CM2.12: } \\
\text { GUC0_D3_5 }\end{array}$ \\
\hline Universidad Granada & $\begin{array}{l}\text { GUGR_D2_1 } \\
\text { C9: GUGR_D3_1, CG36: GUGR_D3_2 }\end{array}$ & $\begin{array}{l}\text { GUGR_D2_2 } \\
\text { C9: GUGR_D3_1,CG36: GUGR_D3_2, } \\
\text { CDM3.4: GUGR_D3_3, CDM4.5: } \\
\text { GUGR_D3_4 }\end{array}$ \\
\hline Universidad Huelva & $\begin{array}{l}\text { GUH_D1_1, GUH_D1_2 } \\
\text { E29: GUH_D3_1,CT6: GUH_D3_2 }\end{array}$ & $\begin{array}{l}\text { GUH_D2_1 } \\
\text { E29: GUH_D3_1, CT6: GUH_D3_2, } \\
\text { E23: GUH_D3_3, G13: GUH_D3_4 }\end{array}$ \\
\hline Universidad Jaén & $\begin{array}{l}\text { CFB23: GUJ_D3_1, CFBDD5: } \\
\text { GUJ_D3_2 }\end{array}$ & CFB23: GUJ_D3_1, \\
\hline Universidad Málaga & $\begin{array}{l}\text { GUM_D1_1 y GUM_D1_2: } \\
\text { Relacionados con las competencias } \\
\text { CE29: GUM_D3_1, CE414: } \\
\text { GUM_D3_2 } \\
\text { GUM_D4_1 } \\
\text { GUM_D5_1 }\end{array}$ & $\begin{array}{l}\text { GUM_D1_1 y GUM_D1_3: } \\
\text { Relacionados con las competencias } \\
\text { CE29: GUM_D3_1, CE332: } \\
\text { GUM_D3_3 } \\
\text { GUM_D4_1 } \\
\text { GUM_D5_1 }\end{array}$ \\
\hline Universidad Sevilla & $\begin{array}{l}\text { GUS_D1_1 } \\
\text { GUS_D2_1 } \\
\text { EP15: GUS_D3_1, M28: GUS_D3_2 }\end{array}$ & $\begin{array}{l}\text { GUS_D1_2 } \\
\text { EP15: GUS_D3_1, M22: GUS_D3_3 } \\
\text { GUS_D5_1 }\end{array}$ \\
\hline
\end{tabular}

Después de analizar los planes de estudio de las ocho universidades es llamativo que si bien en todas están presentes las competencias en Sostenibilidad en un nivel significativo (Nivel 3), esta presencia tiene un reflejo muy débil en los demás elementos de la planificación docente. Se aprecian algunas referencias hacia los objetivos y contenidos, que solo alcanzan un cierto nivel de relevancia, de forma alternativa, en algunas de las universidades.

Es muy significativo del nivel de consideración de la necesidad real de integrar la Sostenibilidad en las aulas universitarias, la ausencia absoluta de consideración alguna en los aspectos metodológicos y casi absolutos (solo una universidad incluye alguna referencia) sobre la evaluación. Son precisamente dos elementos determinantes para el desarrollo de los planes de estudio y su concreción en las aulas de formación de maestros. 
Ya que, como indicábamos al principio, integrar la Sostenibilidad en el ámbito de la formación de maestros, no se trata solo de incluir competencias ni contenidos ambientales en los currículums, sino también de proporcionar un cambio de mentalidad en el alumnado, para ello es necesario promover estrategias metodológicas adecuadas (Cardeñoso et al., 2013). Significa proponer estrategias que partan de trabajar problemas cercanos al alumnado y a la sociedad, asumiendo que los procesos de enseñanza-aprendizaje se lleven a cabo desde la cooperación y el trabajo en equipo, así como la toma de decisiones colectiva y los distintos puntos de vista. En el mismo sentido, promover procesos evaluativos que reflejen un seguimiento del proceso tanto de la enseñanza como del aprendizaje que desarrolla en el aula.

Es difícil pensar que estos planes de estudios promuevan realmente una integración de la Sostenibilidad en las aulas de formación, es necesario un proceso de revisión importante para avanzar en dicha integración que favorezca una formación de los maestros más adecuada a la realidad en la que nos movemos.

El estudio realizado podría compararse con el de Aznar Minguet \& Ull Solís (2019), en el que se hace un análisis documental en los planes de estudio y guías docentes de distintos grados en la Universidad de Valencia. Entre los grados analizados estaban el Grado en Educación Infantil y el Grado en Educación Primaria. El objetivo primero era estudiar la situación inicial de los programas de las asignaturas en relación a la Sostenibilidad, lo que permitiría conocer posteriormente el grado de ambientalización curricular de los grados seleccionados para diagnosticar la situación de partida, y así reformular y orientar los programas curriculares en las titulaciones mencionadas.

Como pequeña reflexión con respecto a los resultados de nuestro estudio, cabría destacar que sostenibilizar el currículum es una tarea colectiva que involucra a distintos miembros de la comunidad universitaria, teniendo claro los aspectos que se quieren cambiar y las asignaturas que necesitarían un punto de vista más enfocado a la Sostenibilidad.

El análisis de las Memorias que hemos realizado será el punto de partida para una futura investigación relacionada con la inclusión de la Sostenibilidad en el Grado de Educación Primaria, algo fundamental en estos tiempos en el que nuestro planeta nos pide soluciones urgentes debido a la emergencia climática que nos afecta a todos los ciudadanos, siendo relevante involucrar a la población infantil, ya que son los que sufrirán las consecuencias futuras si no se empiezan a solventar los problemas en el presente.

Para ello, es necesario que los futuros maestros, aquellos que serán los formadores de los más pequeños, comiencen a crear una visión más amplia sobre la Sostenibilidad desde que están en la Universidad, reiterando una vez más la importancia de esta institución en el proceso de sostenibilización curricular y de formar a expertos. La comprensión y la implicación en el aula de la Sostenibilidad son esenciales para la conexión entre las ideas previas de los maestros, y así fomentar la integración y la relación con los principios de la ES (García-González et al., 2020b). 


\section{Conclusiones}

Esta investigación nos ha ayudado a responder a las cuestiones que nos planteábamos al inicio del estudio. En primer lugar, con respecto a la pregunta sobre si los Grados de Educación Primaria de las universidades andaluzas incluyen aspectos relacionados con la Sostenibilidad en sus Memorias, la respuesta es que sí, aunque la presencia de la misma difiere entre universidades, tal como se puede apreciar en los de resultados. En el caso de los objetivos, en Cádiz, Huelva, Málaga y Sevilla sí se incluye la Sostenibilidad en los Niveles 1 y 3 , mientras que el resto de universidades se sitúan en el Nivel 0.

Con respecto a los contenidos, las universidades de Jaén y Málaga no incluyen referencias a la Sostenibilidad en sus asignaturas, mientras que en las demás universidades encontramos variedad entre los Niveles 1 y 3.

En cuanto a las competencias, las ocho universidades se establecen en el máximo nivel, pues en todas ellas aparece la Sostenibilidad en diversas competencias en forma de conocimientos, habilidades, actitudes y destrezas en asignaturas tanto de didáctica de las ciencias experimentales como didáctica de las ciencias sociales y didáctica de la lengua.

Por otra parte, en lo que respecta a la metodología, todas las universidades están clasificadas en el Nivel 0 por no presentar actividades metodológicas que promuevan la integración de la Sostenibilidad.

Y, por último, los sistemas de evaluación de la mayoría de las universidades se establecen en un Nivel 0, ya que no se considera entre sus criterios los logros en materia de Sostenibilidad, exceptuando a Sevilla, clasificada en un Nivel 2 al tener un criterio de evaluación en una asignatura que sí evalúa la Sostenibilidad.

En resumidas cuentas, podríamos decir que la Sostenibilidad en las universidades andaluzas solo estaría presente en algún nivel en los objetivos, contenidos y competencias.

De acuerdo con la segunda pregunta que nos planteábamos al inicio del estudio, los aspectos que aparecen relacionados con la Sostenibilidad dentro de los objetivos, contenidos y competencias, están basados en valores, principios y conocimientos que capacitan a los estudiantes para sentir, pensar y actuar sobre el mundo. Del mismo modo, se mencionan comportamientos éticos como la responsabilidad, así como la capacidad de toma de decisiones frente a la problemática ambiental. Todo ello girando en torno a lo social, a lo económico y a lo ambiental.

En lo que se refiere a los objetivos, competencias y contenidos, aparece la Sostenibilidad desde distintos enfoques, dependiendo de la asignatura curricular en la que se encuentren, habiendo diferencias entre las asignaturas de didáctica de las ciencias experimentales y didáctica de las ciencias sociales o de la lengua, que son las que se han encontrado.

Ahondando en la cuestión que nos planteábamos sobre las competencias, encontramos similitudes en aquellas que aparecen repetidas veces en distintas universidades. Estas competencias reflejan la Sostenibilidad tanto de manera transversal como de forma explícita en asignaturas tanto de didáctica de las ciencias experimentales como en didáctica de las ciencias sociales. 
En relación con la cuestión que atañe a los objetivos, los que podemos encontrar en las memorias son diversos, dependiendo de la procedencia con respecto a asignaturas que forman parte de didáctica de las ciencias experimentales o, en su contra, de las que pertenecen a la didáctica de las ciencias sociales.

Seguidamente, los contenidos que hacen referencia a la Sostenibilidad en las memorias difieren, puesto que las asignaturas donde aparecen pertenecen o bien a la didáctica de las ciencias experimentales, o bien a didáctica de ciencias sociales o de la lengua. Independientemente de esta razón, aparece la Sostenibilidad como contenido de asignaturas en seis de las ocho universidades analizadas, estando exentas Jaén y Málaga.

Sin embargo, los objetivos, contenidos y competencias mencionados no quedan reflejados explícitamente en las metodologías propuestas, ni tampoco en la evaluación de las asignaturas. De ahí la razón por la que se han establecido las dimensiones que corresponden a la metodología y a los sistemas de evaluación en el nivel más bajo de nuestro sistema de análisis. Solo la Universidad de Sevilla hace referencia a la Sostenibilidad en los sistemas de evaluación, curiosamente en la asignatura de Didáctica de las Matemáticas.

Tras todo lo recopilado hasta el momento, queda patente que la Sostenibilidad está todavía lejos de integrarse de una manera más profunda en las Memorias de los grados analizados y, en consecuencia, en las aulas, considerando, además, que no solamente ha de integrarse en el terreno de la formación de maestros, sino que ha de incluirse en la formación de futuros profesionales en general.

La Sostenibilidad Curricular implica el desarrollo de una serie de estrategias, principios y valores establecidos en un plan de estudios que pretende formar para una profesión. La universidad, como institución, no debe estar ajena a este proceso, pues es considerada un motor de cambio social. Analizar la Sostenibilidad en las Memorias de Grado es solo el primer paso para analizar y reflexionar sobre cómo conseguir el cambio.

Aunque las Memorias reflejen la inclusión de la Sostenibilidad en objetivos, contenidos y competencias, está claro que no es suficiente, pues los docentes de la propia universidad deben estar preparados para comprenderla y trabajarla en todos sus aspectos, de modo que puedan impartirla y transmitirlas a su alumnado durante su periodo de formación.

\section{Agradecimientos}

Esta investigación fue financiada por el Proyecto "Sostenibilidad en Educación Superior: Evaluación del alcance de la Agenda 2030 en la innovación curricular y el desarrollo profesional docente en las Universidades Andaluzas" (B-SEJ-424-UGR18), en el marco del Programa Operativo FEDER-Andalucía 2014-2020.

\section{Referencias bibliográficas}

Albareda-Tiana, S. \& Gonzalvo, M. (2013). Competencias genéricas en sostenibilidad en la educación superior. Revisión y compilación. Revista de Comunicación de la SEECI, XV(32), 141-159. https://doi.org/10.15198/seeci.2013.32.141-159

Álvarez, O. C. (2004). Educación ambiental a partir de tres enfoques: comunitario, sistémico e interdisciplinario. Revista iberoamericana de educación, 35(1), 1-7. 
Azcárate, P., Navarrete, A., \& García-González, E. (2012). Aproximación al nivel de inclusión de la sostenibilidad en los curricula universitarios. Profesorado. Revista de currículum y Formación del Profesorado, 16(2), 105-119

Aznar Minguet, P., \& Ull Solís, A. (2019). Educación y Sostenibilidad en la Universidad de Valencia: construyendo futuro desde el pasado. Revista de Educación $\begin{array}{llll}\text { Ambiental } & y & \text { Sostenibilidad } & 1202 .\end{array}$ http://dx.doi.org/10.25267/Rev_educ_ambient_sostenibilidad.2019.v1.i1.1202

Barrón, Á., Ferrer-Balas, D., \& Navarrete Salvador, A. (2010). Sostenibilización curricular en las universidades españolas. ¿Ha llegado la hora de actuar? Revista Eureka sobre Enseñanza y Divulgación de las Ciencias, 7(no extraordinario), 388-399. Disponible en https://www.redalyc.org/articulo.oa? id $=92013009018$

C.A.D.E.P.-C.R.U.E. (2012). Directrices para la introducción de la Sostenibilidad en el Curriculum. Actualización de la declaración institucional aprobada en 2005. (Consultado el 22 enero 2021) Recuperado de: http://www.crue.org/Documentos compartidos/Declaraciones/Directrices_Sosteniblidad_Crue2012.pdf

Cardeñoso, J. M., Azcárate, P., \& Oliva, J. M. (2013). La inclusión de la sostenibilidad en la formación inicial del profesorado de Secundaria de Ciencias y Matemáticas. Revista Eureka sobre Enseñanza y Divulgación de las Ciencias, $10\left(\mathrm{n}^{\mathrm{o}}\right.$ extraordinario), 780-796. http://dx.doi.org/10.25267/Rev_Eureka_ensen_divulg_cienc.2013.v10.iextra.1 9

Cebrián, G., \& Junyent, M. (2014). Competencias profesionales en Educación para la Sostenibilidad: un estudio exploratorio de la visión de futuros maestros. Enseñanza de las ciencias: revista de investigación y experiencias didácticas, 32(1), 29-49. https://doi.org/10.5565/rev/ensciencias.877

De la Rosa, D., Giménez, P., \& De la Calle, C. (2019). Educación para el desarrollo sostenible: El papel de la universidad en la agenda 2030. Transformación y diseño de nuevos aprendizajes. Prisma Social, (25), 179-202.

Denzin, N. K., \& Lincoln, Y. S. (2005). The Sage Handbook of Qualitative Research (3. ed.). Sage.

Erice, M. (2004). Las competencias ambientales en la formación profesional en Educación Ambiental. En Organismo Autónomo Parques Nacionales, Nuevas tendencias en investigación en Educación Ambiental, (pp. 281-297). Ministerio de Medio Ambiente.

García-González, E., Jiménez-Fontana, R., \& Azcárate, P. (2020a). Education for Sustainability and the Sustainable Development Goals: Pre-Service Teachers' $\begin{array}{llll}\text { Perceptions and Knowledge. Sustainability } & \text { 12:7741. }\end{array}$ https://doi.org/10.3390/su12187741 
García-González, E., Jiménez-Fontana, R., \& Azcárate, P. (2020b). Approaches to teaching and learning for sustainability: Characterizing students' perceptions. Journal of Cleaner Production, 274, 122928.

Geli, M., Collazo, L., \& Mulà, I. (2019). Contexto y evolución de la sostenibilidad en el curriculum de la universidad española. Revista de Educación Ambiental y Sostenibilidad $1(1)$ 1102. https://doi.org/10.25267/Rev_educ_ambient_sostenibilidad.2019.v1.i1.1102

Gómez, D., \& Roquet, J. (2009). Metodología de la investigación. Universitat Oberta de Catalunya.

Guimarães, S. S. M., \& Tommasiello, M. G. C. (2007). Las ideas de sostenibilidad de los alumnos de un curso de biología. Revista Electrónica de Enseñanza de las Ciencias, 6(1). Disponible en http://reec.uvigo.es/volumenes/volumen6/ART13_Vol6_N1.pdf

Hopkins, C. \& Ravindranath, M.J. (2007). Teacher Education - a crucial contribution to DESD. Re-commendations. International Conference on DESD, CEE (Ahmedabad, November 2007).

Ley Orgánica 8/2013 para la mejora de la calidad educativa (LOMCE). Boletín Oficial del Estado, 10 de diciembre de 2013, número 295.

López Noguero, F. (2002). El análisis de contenido como método de investigación. XXI. Revista de educación, (4),167-180.

Macedo, B. (2005). Educación para la Sostenibilidad. (Consultado el 18 de enero de 2021).

Recuperado

de

https://unesdoc.unesco.org/ark:/48223/pf0000162178?

posInSet=1\&queryId=79620b75-12e6-49ea-9b32-efae8e41d6ae

Murga-Menoyo, M. A., \& Bautista-Cerro, M. J. (Edas.) (2019). Guía PRADO. Sostenibilizar el currículo de la Educación Secundaria. UNED. https://doi.org/10.5944/catedra.eads.50112

Orr D. W. (2004). Earth in mind: on education, environment, and the human prospect. Island Press. https://doi.org/10.1177/108602669600900421

Poza-Vilches, F., García-González, E., Solís-Espallargas, C., Velasco-Martínez, L. C., López-Alcarria, A., Estrada-Vidal, L. I., Jiménez-Fontana, R., Rodríguez-Marín, F., Puig-Gutiérrez, M., Tójar Hurtado, J. C. \& Gutiérrez-Pérez, J. (2021). Greening of the syllabus in faculties of education sciences through sustainable development goals: the case of public Andalusian universities (Spain). International Journal of Sustainability in Higher Education. https://doi.org/10.1108/IJSHE-02-20210046

Ruiz-Rico, C. (2016). La responsabilidad social como estrategia de innovación docente universitaria: objetivos y metodología de una educación sostenible. Revista Jurídica de Investigación e Innovación Educativa (REJIE Nueva Época), (13), 917.

Sachs, J., \& Vernis, R. (2015). La era del desarrollo sostenible. Deusto.

Torres-Porras, J., \& Arrebola, J. C. (2018) Construyendo la ciudad sostenible en el Grado de Educación Primaria. Revista Eureka sobre Enseñanza y Divulgación de 
las

Ciencias

15(2),

2501.

https://doi.org/10.25267/Rev_Eureka_ensen_divulg_cienc.2018.v15.i2.2501

UNESCO (2017a). Educación para los Objetivos de Desarrollo Sostenible. Objetivos de Aprendizaje. (Consultado el 23 de enero de 2021). Recuperado de https://unesdoc.unesco.org/ark:/48223/pf0000252423?

posInSet=1\&queryId=e80d81ed-c328-4c6f-a9c6-613caaa35852

UNESCO. (2017b). Orientaciones sobre la ciencia de la sostenibilidad en la investigación y la educación. (Consultado el 10 de enero de 2021). Recuperado de https://unesdoc.unesco.org/ark:/48223/pf0000260600_spa? posInSet=1\&queryId=26880197-2053-45b4-b131-4247e2521a65

Vilches, A., \& Gil, D. (2012). La Educación para la Sostenibilidad en la Universidad: el reto de la formación del profesorado. Profesorado. Revista de currículum y formación del profesorado, 16(2), 25-4 